\title{
A Numerical Study of Depth of Penetration of Eddy Currents
}

\author{
S.Majidnia*a,b , R.Nilavalan ${ }^{b}$, J. Rudlin ${ }^{a}$ \\ a. TWI Ltd, Cambridge ,United Kingdom \\ ${ }^{b}$ Brunel University, London ,United Kingdom \\ shiva.majidnia@twi.co.uk
}

\begin{abstract}
The simple standard depth of penetration equation used for most eddy current calculations does not take into account the effects of the size and type of the coil, the lift-off or the specimen thickness. The work described in this paper includes a study of the depth of penetration of eddy currents for different coil sizes including surface and encircling type coils by taking advantage of the sophisticated finite element modelling software COMSOL. Knowledge of these effects plays an important role in designing eddy current coils and examinations of conductive materials with the eddy current technique. This paper includes a study of the effects of all the dimensions of a surface coil, encircling coil, lift-off and specimen thickness at several excitation frequencies. It is demonstrated from the results obtained from these numerical studies that the coil parameters affect the depth of penetration at different frequencies.
\end{abstract}

Key words: Standard depth of penetration, Eddy current testing, Finite element analysis

\section{Introduction}

Eddy current testing is widely used for the quality assurance of parts made of conducting materials [1]. This method is applicable to surface or near- surface flaw detection because of the decrease in magnetic flux and eddy current density with depth in the material as a result of the skin effect. When a plane electromagnetic wave is incident perpendicularly on a conducting half-space, the skin effect depends only on the wave frequency and the magnetic permeability and electrical conductivity of the material. In this case the depth in the material at which the eddy current density decreases to $1 /$ e $(37 \%)$ of the surface density is called the standard depth of penetration and is commonly used as a criterion for the eddy current inspection process [2]. Following the earlier literature, the term standard depth of penetration will be used in this paper. A real coil working as an eddy current probe does not generate a plane electromagnetic wave. For this reason the standard depth of penetration is a material/test parameter rather than a true measure of penetration. The true depth of penetration, besides depending on frequency, magnetic permeability and electrical conductivity, depends also on the sample thickness, which limits the depth of penetration to its thickness, and the probe dimensions. Mottl [2] studied the relationship between the true depth of penetration and the standard depth of penetration and confirmed that the electromagnetic field generated by the eddy current coil is not a plane 
wave and also depends on the probe diameter and the thickness of the material under test. The study included three different scenarios which were a coil above a conducting half space, an air-cored coil above a conducting sheet of finite thickness and an air-cored coil above a conducting half-space covered with cladding of finite thickness. It was concluded that, for coils with Rs/ $\delta>10$ (where Rs is the mean coil radius) and above a sample of infinite thickness, the depth of penetration calculated from the formula can be accurate. However the study did not quantitatively study the effects of coil parameters and type at different frequencies.

This paper includes the study of the depth of penetration of eddy currents for different coil sizes and type by taking advantage of the sophisticated finite element modelling software COMSOL.

\section{Definition of depth of penetration of eddy currents}

The standard depth of penetration (SDoP) for eddy current testing is usually defined by the graph shown in Figure 1, which is derived from the plane wave equation for the depth of penetration (DoP) as given in Equation 1.

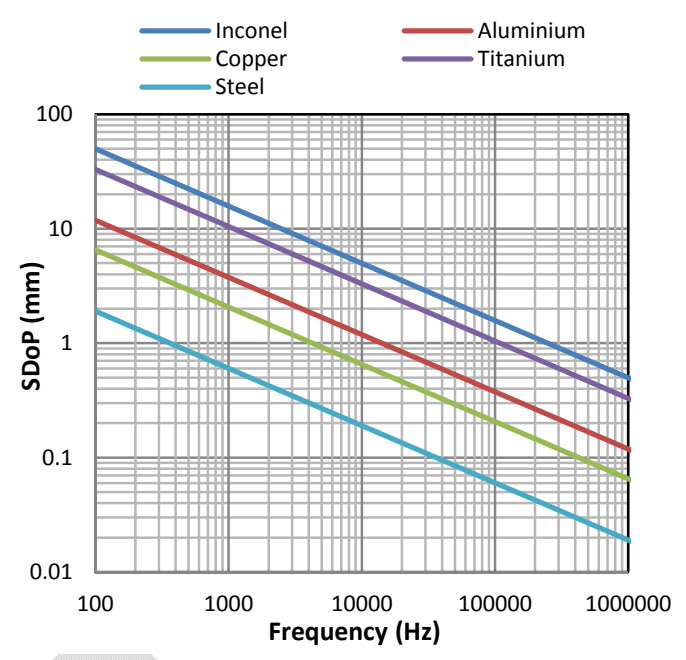

Figure 1. Standard depth of penetration of eddy currents calculated from Equation 1

$\delta=\frac{1}{\sqrt{\pi f \mu \sigma}} \quad, \quad$ Equation 1

where $\delta$ is the standard depth of penetration of the eddy currents in the material $(m), f$ is the excitation frequency $(\mathrm{Hz}), \boldsymbol{\mu}$ is the magnetic permeability $(\mathrm{H} / \mathrm{m})$ of the material under test and $\boldsymbol{\sigma}$ is the electrical conductivity $(\mathrm{S} / \mathrm{m})$ of the material under test.

From Equation 1 the depth in the material at which the eddy current density under the probe coil decreases to $1 /$ e (37\%) of the surface density is calculated. Similarly, the effective depth of penetration is defined as the depth at which the current density decreases to $1 / \mathrm{e}^{3}(4.9 \%)$. It is assumed that any disturbance in the eddy current flow below this depth does not produce impedance changes in the coil, so the effective depth of penetration can be considered as the maximum depth in the material that can be inspected by the eddy current method [2].

\section{Model details}

In order to model the eddy current phenomena as applied in non-destructive testing, the magnetic 
field interface and the Alternating Current /Direct Current (AC/DC) module of COMSOL Multiphysics were used [3]. The magnetic field interface has the equations and external currents for modelling magnetic fields and solving for the magnetic vector potential [4]. Two model scenarios are considered in this paper namely surface and encircling probes. The following sections describe the model details for each scenario and the results.

\section{Surface probes}

An air-cored surface coil is first considered to investigate the effects of probe parameters and examination effects on the depth of penetration of the eddy currents. Schematics of a surface type probe can be found in Figure 2. Due to the axisymmetric nature of these studies a 2D axisymmetric model was developed. This method is effective and computationally feasible. The model consisted of a coil, specimen, and an air domain. The general model geometry can be found in Figure 3.

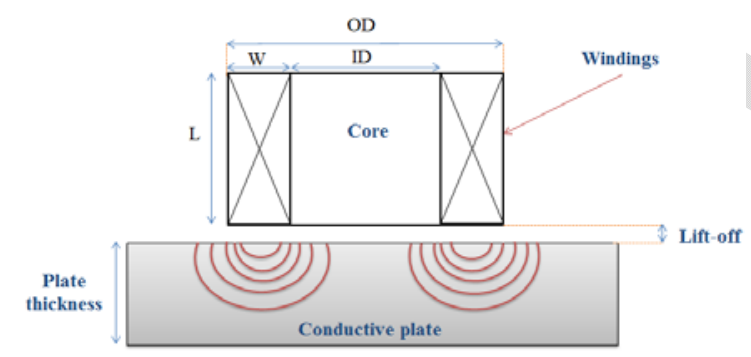

Figure 2. General schematic of a surface probe

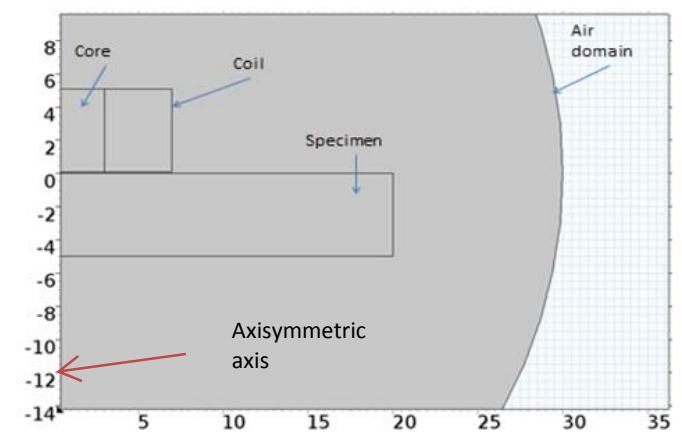

Figure 3. General model geometry
The specimen was considered to be an aluminium plate with conductivity of $3.774 \times 107 \mathrm{~S} / \mathrm{m}$. The core of the coil was considered to be air. A convergence study was carried out on the mesh element size to ensure the mesh size was fine enough to have no effect on the results [5].

\subsubsection{Coil diameter}

First the size of the coil was studied in terms of its inside and outside diameters as labelled Inside Diameter (ID) and Outside Diameter (OD) in Figure 2 accordingly. These studies were completed with 30 turn coils with $5 \mathrm{~mm}$ height. The lift-off, which is described as the distance of the coil from the material, was set to $0.1 \mathrm{~mm}$. The plate was assumed to be $5 \mathrm{~mm}$ thick. Table 1 shows the coil sizes used for these simulations.

Table 1. Coil parameters used in Comsol modelling

\begin{tabular}{cccc} 
Name & OD $(\mathrm{mm})$ & ID $(\mathrm{mm})$ & W(mm) \\
\hline \hline Coil_1 & 2 & 0.5 & 0.5 \\
Coil_2 & 2 & 1 & 1 \\
Coil_3 & 3 & 1 & 2 \\
Coil_4 & 3 & 2 & 1 \\
Coil_5 & 3 & 2.5 & 0.5 \\
Coil_6 & 4 & 1 & 3 \\
Coil_7 & 4 & 2 & 2 \\
Coil_8 & 4 & 3 & 1 \\
Coil_9 & 4 & 3.5 & 0.5 \\
Coil_10 & 5 & 1 & 4 \\
Coil_11 & 5 & 2 & 3 \\
Coil_12 & 5 & 3 & 2 \\
Coil_13 & 5 & 4 & 1 \\
Coil_14 & 6 & 1 & 5 \\
Coil_15 & 6 & 2 & 4 \\
Coil_16 & 6 & 3 & 3 \\
Coil_17 & 6 & 4 & 2 \\
Coil_18 & 6 & 5 & 1 \\
Coil_19 & 7 & 4 & 3
\end{tabular}

The depth at which the eddy current density was reduced to $37 \%$ of its value at the surface of the 
aluminium plate under the conductor was found. The results obtained from these simulations are compared in logarithmic scales with the standard depth of penetration of eddy currents calculated for aluminium using Equation 1, and are shown in Figure 4.

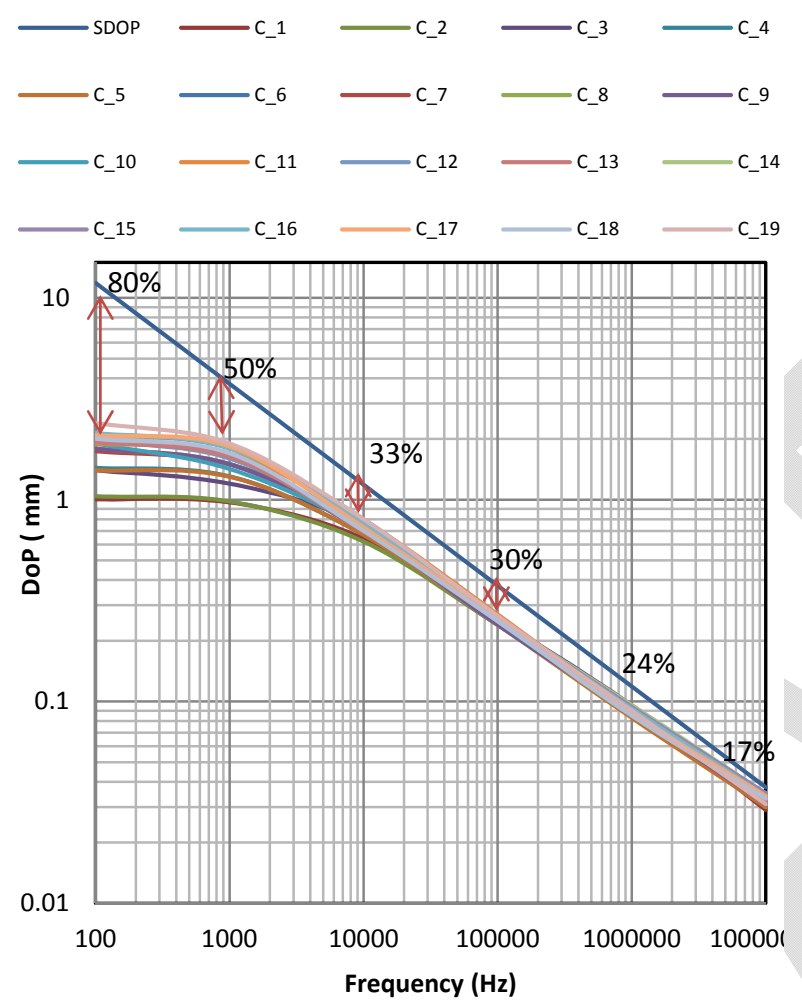

Figure 4. Depth of penetration obtained from the models in Comsol for coil sizes ranging from 2-7 mm OD and effective width from $0.5-3 \mathrm{~mm}$

The results from these simulations show that the depth of penetration of the eddy currents is greatly affected by the size of the coil. For frequencies lower than $10 \mathrm{kHz}$ these differences are much greater than those for frequencies higher than $10 \mathrm{kHz}$ as shown using percentage values between the greatest DoP found from the models and the SDoP from Equation 1. It is realised that the plane wave formula is predominantly and theoretically valid for a plane electromagnetic wave which is incident perpendicularly on a conducting half-space but, as can be concluded from this graph, this equation is a relatively good estimate for the standard depth of penetration for frequencies higher that $10 \mathrm{kHz}$ in a plate $5 \mathrm{~mm}$ thick.

In order to investigate these effects of the depth of penetration of eddy currents induced in the $5 \mathrm{~mm}$ thick aluminium plate, coils with the same width are plotted in the same graph. This means that coils which have the same width (W) are shown in one graph to make the comparison more straightforward. These graphs can be found in Figures 5 - 8.

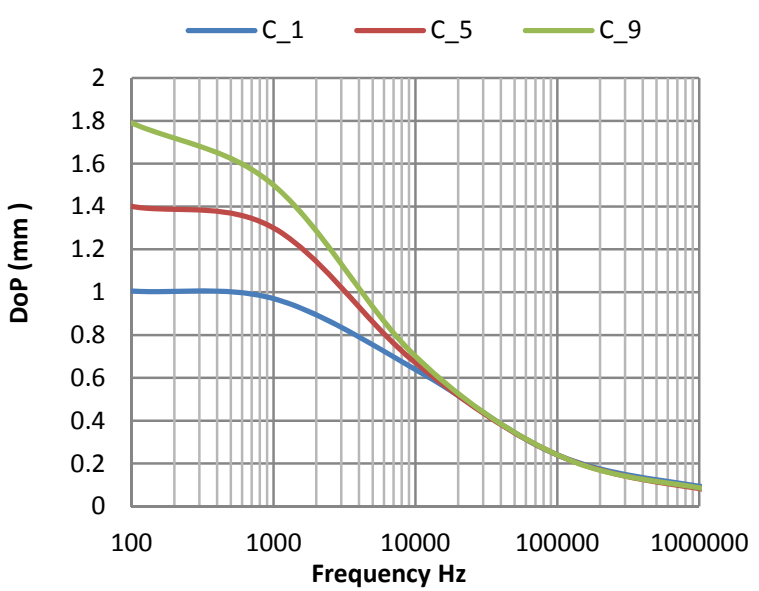

Figure 5. Coils with effective width of $0.5 \mathrm{~mm}$

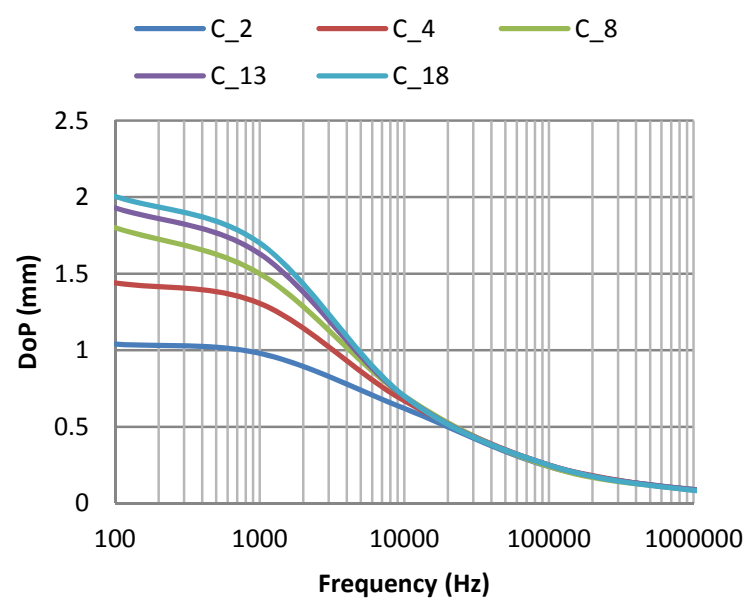

Figure 6. Coils with effective width of $1 \mathrm{~mm}$ 


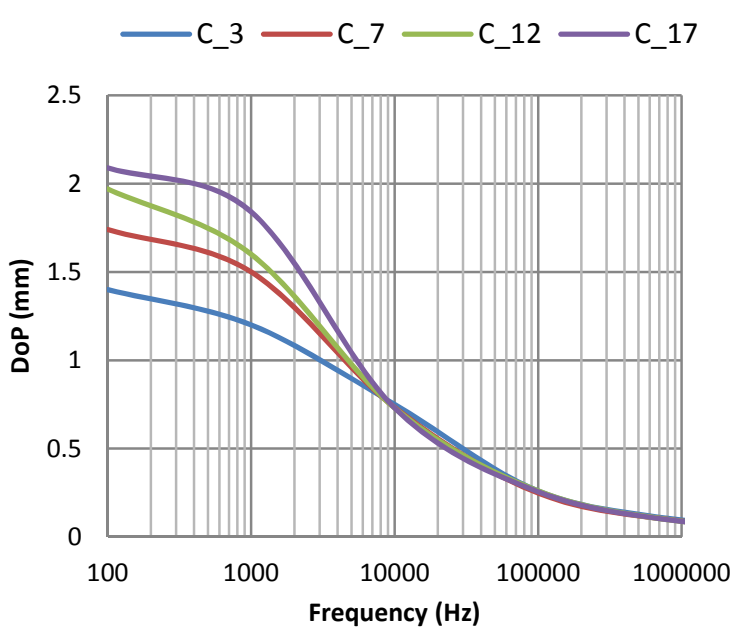

Figure 7. Coils with effective width of $2 \mathrm{~mm}$
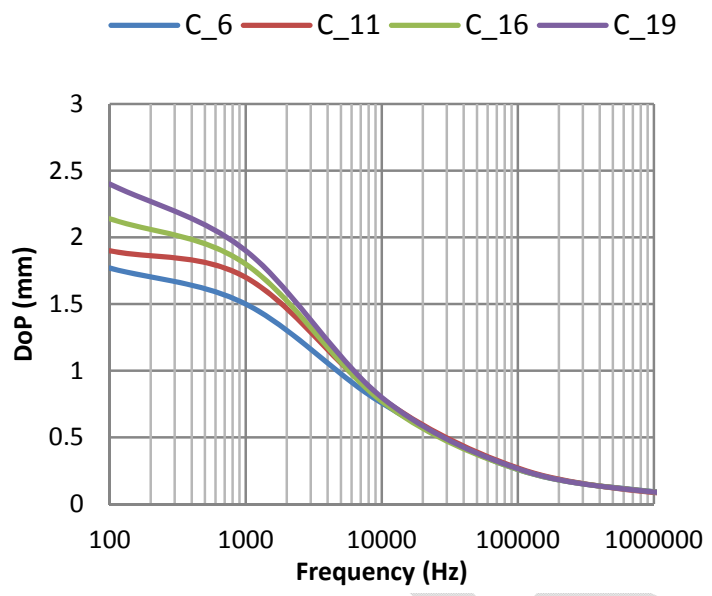

Figure 8. Coils with effective width of $3 \mathrm{~mm}$

Based on the simulation results shown in the graphs of Figures $5-8$ for frequencies ranging between 100 and $10 \mathrm{kHz}$, the coil width has an effect on the depth of penetration. It is also is apparent that for coils with the same width within this range, larger coils have a greater depth of penetration.

\subsubsection{Number of turns}

The effect of the number of turns of the coil on the depth of penetration is studied in this section. These studies were carried out for coil 19 from Table 1 which had the largest diameter and coil width among those studied. The number of turns of the coil ranged from 30 to 300 in 10 turn steps. The results obtained from these simulations revealed that the depth of penetration of the eddy currents does not change with the number of turns for the same width, as can be seen in Figure 9. Although the magnetic field generated by the coil is stronger for a greater number of turns, the depth of penetration by definition is not affected by the number of turns of the coil. This phenomenon should not be misinterpreted as a greater depth of penetration, as only greater sensitivity is achieved by using a greater number of turns. The same statement can be applied to a larger current input to the coil, which will in turn result in a greater primary field generated by the driver coil. It is however recognised that the electrical characteristics of the driver equipment and the pick-up element sensitivity range can be the deciding factors for the number of turns and the current input to obtain the sensitivity needed.

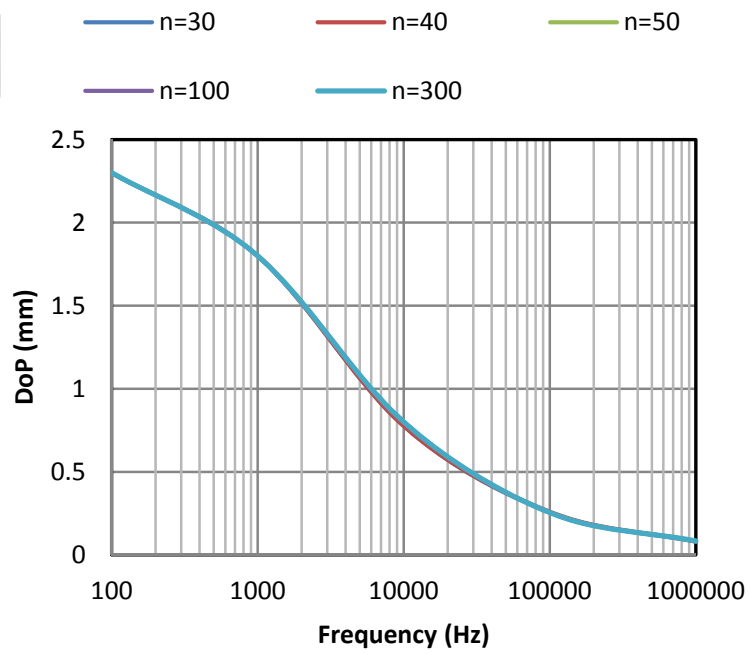

Figure 9. Depth of penetration for coils with different numbers of turns

\subsubsection{Coil height}

In the next stage of these studies, the effect of the height of the coil is investigated. Coil 19 from Table 
1 was chosen and its height was studied for a range of 1 to $10 \mathrm{~mm}$. The simulation results are shown in Figure 10. It is observed that the depth of penetration increases as the height of the coil increases. Although the density of the magnetic field decreases at the surface of the material, meaning that the sensitivity is lower for the same number of turns or current input, the depth of penetration increases with an increase in height for frequencies lower than $3 \mathrm{kHz}$. Percentage comparisons between the DoP of the largest and the smallest height $(H)$ are shown in Figure 10.

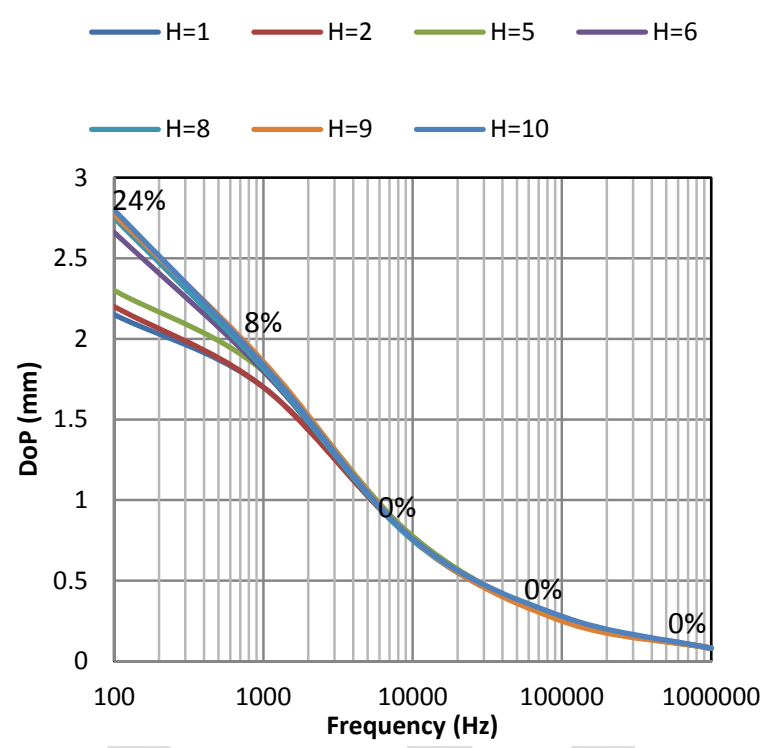

Figure 10. Depth of penetration for coils with various heights

\subsubsection{Lift-off}

Lift-off is described as the distance of the coil from the material under test. It is known that increasing the lift-off reduces the strength of the field at the surface of the material [6]. The simulations were carried out for coil 19 on a $5 \mathrm{~mm}$ thick aluminium plate and the results for lift-off values of $0.1,3,5$ and $10 \mathrm{~mm}$ can be found in Figure 11. The results obtained show that although it might be assumed that the depth of penetration would decrease with increasing lift-off, for frequencies lower than $3 \mathrm{kHz}$ the lift-off increases the depth of penetration as described by the definition, while frequencies higher than $3 \mathrm{kHz}$ are not affected. Percentage comparisons between the greatest and smallest DoP found using the models are also shown in Figure 11. The effect of the eddy current field is however reduced at the receiver element (whether it is a pick-up coil or a magnetic field sensing module) due to the distance of the receiving module from the magnetic field.

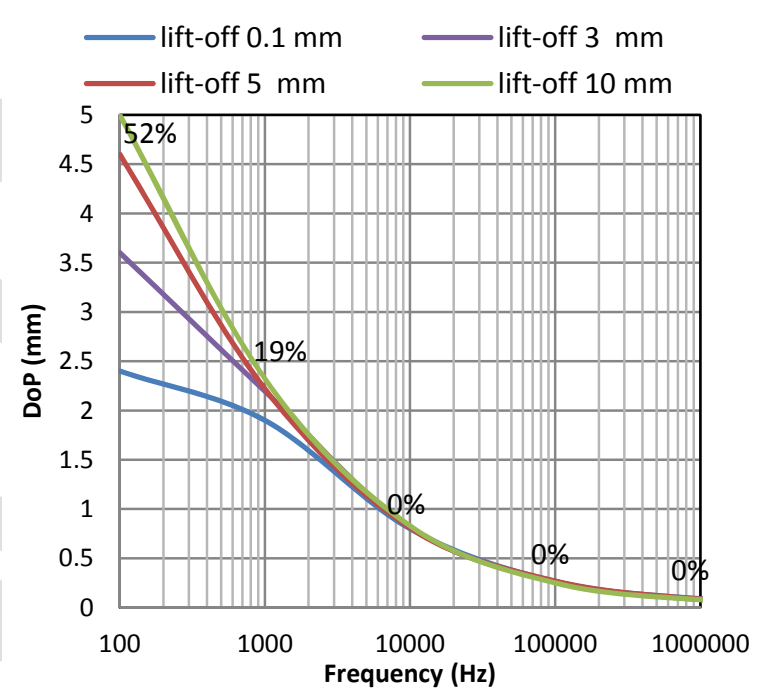

Figure 11. Depth of penetration for different lift-off values

\subsubsection{Sample thickness}

The thickness of the material under test is important in depth of penetration calculations, because the plane wave formula is applied to a conductive half space. The effects of the thickness of the specimen can be studied using FEM. These calculations were carried out for coil 19 with a liftoff of $0.1 \mathrm{~mm}$ and the thickness of the specimen was set at values of $1,2,5,8,10$ and $20 \mathrm{~mm}$. The results obtained from the simulations can be found in Figure 12 and show that depth of penetration is greater in thicker plates for frequencies lower than $10 \mathrm{kHz}$. For frequencies ranging from 100 to 10 $\mathrm{kHz}$, increasing the plate thickness to more than 10 
$\mathrm{mm}$ did not change the penetration depth since the eddy currents are concentrated on the surface of the conductive material.

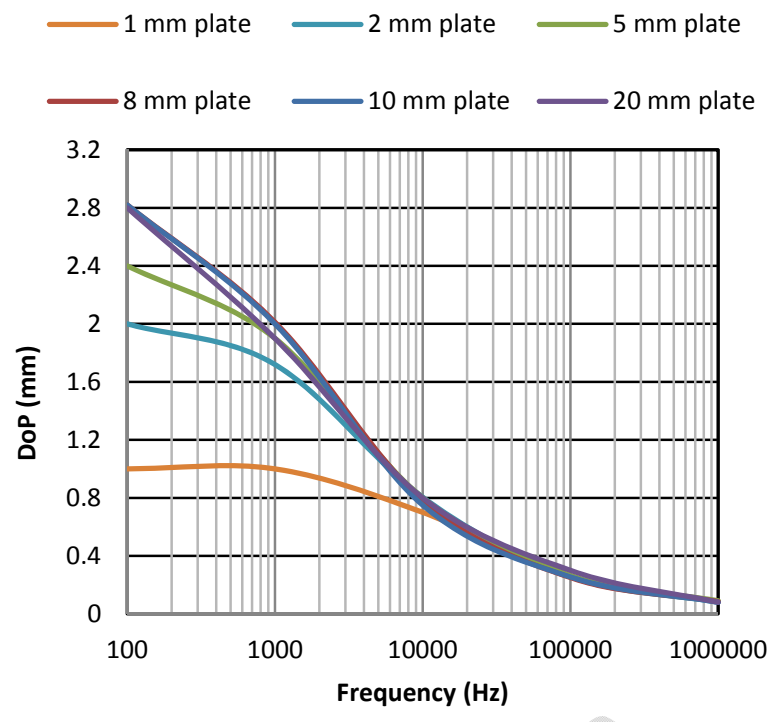

Figure 12. Depth of penetration at specimens with different thicknesses

\subsubsection{Ferrite core}

Ferrite rods are often used as a core for eddy current coils. The use of a ferrite rod enhances the performance of an eddy current coil because it concentrates the lateral spread of the magnetic field generated by the coil [7]. Ferrite cores are usually made of material with high magnetic permeability and low electrical conductivity. The effect of the permeability of the ferrite coil on the depth of penetration is studied here. Coil 19 from Table 1 is used and the air core is replaced by a material that has a conductivity of $1 \mathrm{~S} / \mathrm{m}$ and a relative magnetic permeability of $200,500,700$ and 1500. The plate has a thickness of $5 \mathrm{~mm}$ and the lift-off was $0.1 \mathrm{~mm}$.

The results calculated for the depth of penetration are presented in Figure 13 and show that the ferrite core has increased the depth of penetration compared to its equivalent air-core coil for frequencies lower than $3 \mathrm{KHz}$. A ferrite core also increases the impedance of the coil due to its high permeability. The relative permeability of the ferrite coil has not affected the depth of penetration.

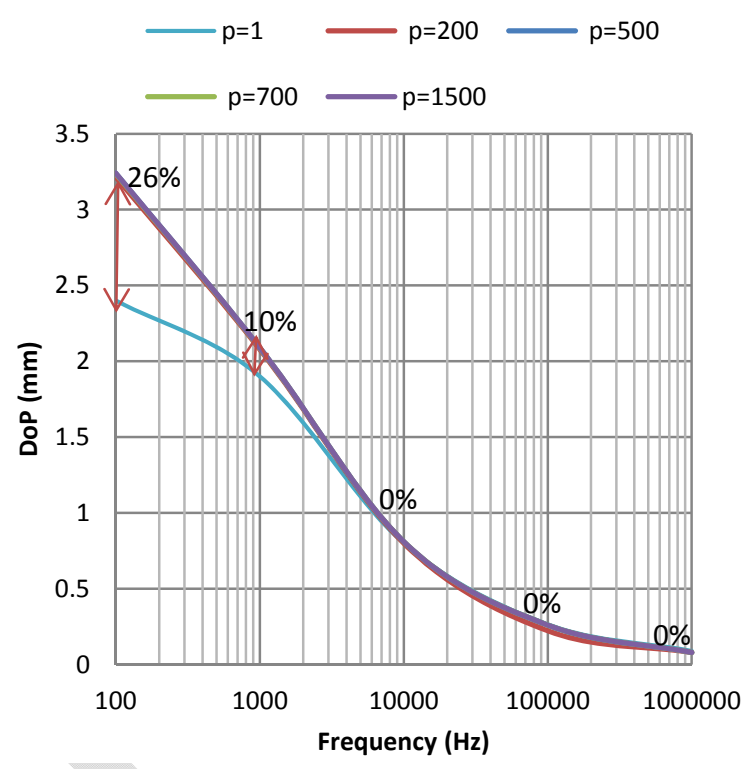

Figure 13. Depth of penetration for ferrite cored and aircored coils

\section{Encircling probe}

All the simulations done up to this stage were on surface type probes. It is important to recognise the effect of the type of the coil on the depth of penetration. Figure 14 shows a general schematic of an encircling probe around a pipe. A 2D axisymmetric model was developed to investigate the depth of penetration of the eddy currents in the pipe wall for an encircling coil around an aluminium pipe. The modelling plane is the rzplane; the horizontal axis represents the r-axis, and the vertical axis represents the z-axis. In this plane, the tube appears as a rectangle and the coil as a circle. To obtain the actual 3D geometry, the 2D axisymmetric geometry revolves about the z-axis. The general geometry of the model used can be found in Figure 15. The model geometry consisted of 3 domains which were the pipe, coil and air domains. The pipe material was set to aluminium 
with the same material properties and had a wall thickness of $5 \mathrm{~mm}$. The lift-off distance was set to $0.5 \mathrm{~mm}$ for this section. The standard depth of penetration in the pipe wall was found at the depth where the eddy current density was reduced to $37 \%$ of its value at the surface of the pipe wall and directly under the centre of the conductor. Coils with 20 turns were considered and their dimensions can be found in Table 2 .

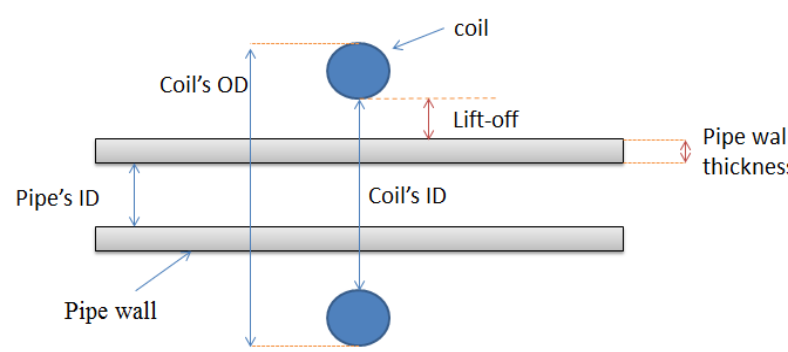

Figure 14. A general schematic of an encircling probe

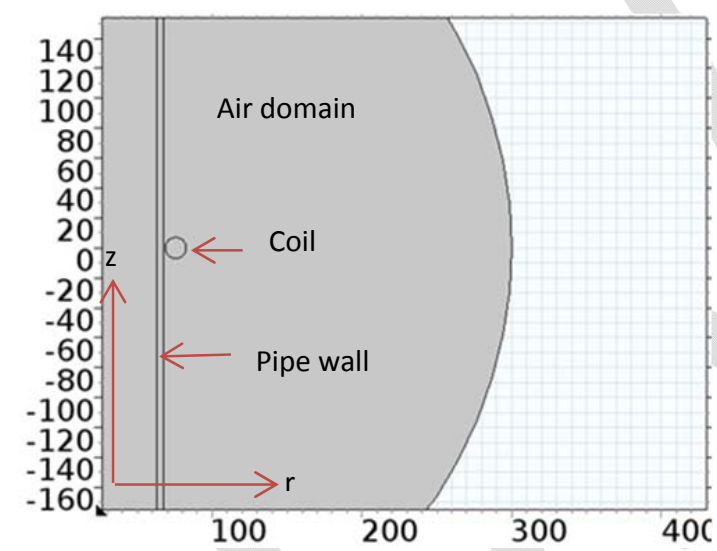

Figure 15. General geometry of the encircling coil used for modelling

Table 2. Encircling coil dimensions (in $\mathrm{mm}$ )

\begin{tabular}{cccc} 
Coil & $\begin{array}{c}\text { Inside } \\
\text { Diameter }\end{array}$ & $\begin{array}{c}\text { Outside } \\
\text { Diameter }\end{array}$ & $\begin{array}{c}\text { Cross Section } \\
\text { Diameter }\end{array}$ \\
\hline \hline 1 & 137 & 165 & 14 \\
2 & 187 & 215 & 14 \\
3 & 237 & 265 & 14 \\
4 & 137 & 177 & 20
\end{tabular}

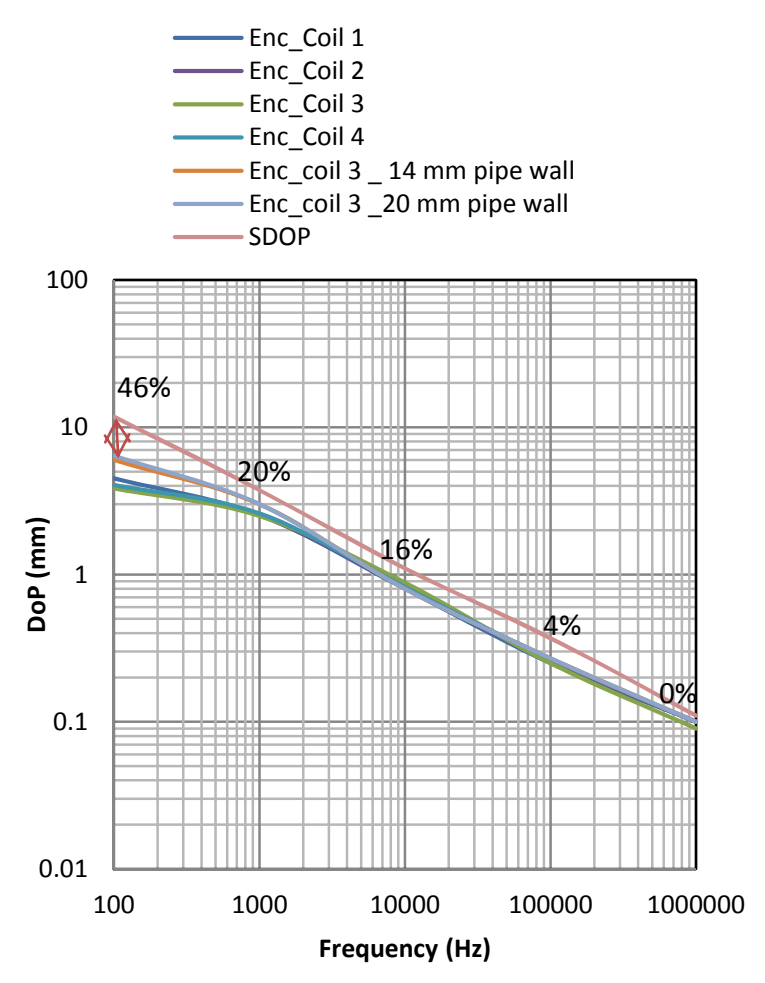

Figure 16. Depth of penetration of eddy currents for encircling coils

The simulation results presented in Figure 16 show that changes to the size of the coil do not affect the standard depth of penetration by a great extent for the proposed encircling coils; however the results from the encircling coils show a greater depth of penetration compared to the surface type coils studied. Since for lower frequencies the eddy currents nearly penetrate the wall thickness of 5 $\mathrm{mm}$, the pipe wall thickness was increased to 14 and $20 \mathrm{~mm}$ to investigate the real depth of penetration on a thicker pipe wall. For this purpose coil 3 from Table 2 was considered with the same lift-off. The results of these scenarios are also presented in Figure 16. The percentage comparisons between the greatest DoP found from the simulation and the one calculated from Equation 1 are also shown in Figure 16. 


\subsubsection{Lift-off}

Since it was found that the depths of penetration for lower frequencies were higher than the ones calculated for the surface type probes, it is beneficial to study the depth of penetration of an encircling coil when there is a larger lift-off value. For these investigations, the aluminium pipe had a wall thickness of $30 \mathrm{~mm}$ to allow for the possible increased depth of penetration and coil 3 from Table 2 was chosen.

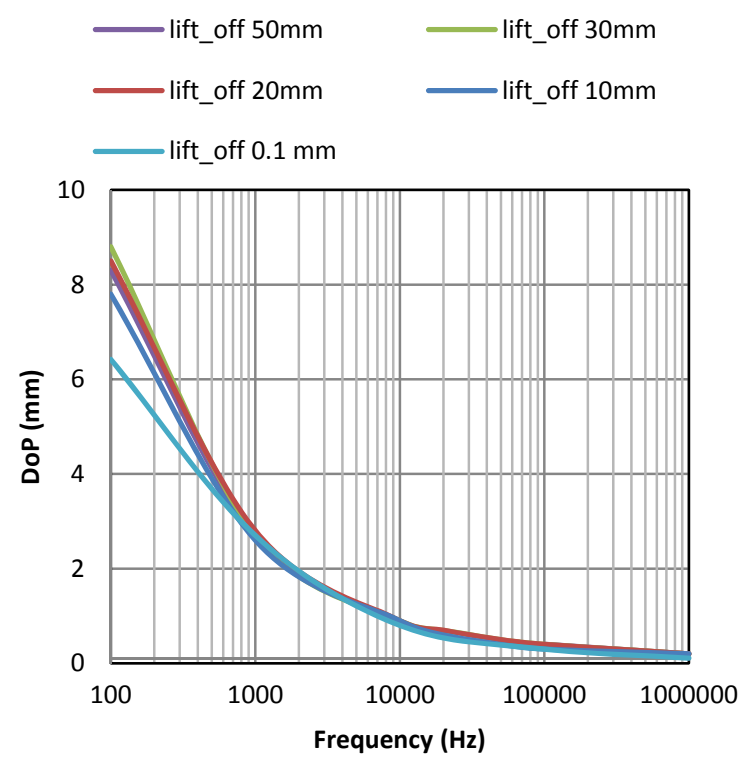

Figure 17. Depth of penetration for encircling coils for different lift-off values

The simulation results are shown in Figure 17. It is observed that the depth of penetration for frequencies lower than $500 \mathrm{~Hz}$ is affected by the lift off. It should be noted that although the depth of penetration is found by the method explained earlier, the strength of the field is greatly reduced which in turn result in lower sensitivity. When compared to lift-off effects on the DoP of surface probes, encircling probes are affected at lower frequencies.

\section{Summary}

In this paper a numerical study of the depth of penetration of the eddy currents in aluminium plate and pipe has been reported. This included a study of the depth of penetration using surface probes and encircling probes. The investigations were carried out to study the effects of the size of a surface probe, the number of turns on the coil, the lift-off values and the plate and wall thicknesses.

From the investigations, it was confirmed that the standard depth of penetration of an eddy current coil, besides depending on the electromagnetic wave frequency and the magnetic permeability and electrical conductivity of the sample, also depends strongly on the type of the coil, its dimensions, the sample thickness and the lift-off.

Comparisons of the results show that different parameters affect the standard depth of penetration of the eddy currents at different frequencies. This information is important in the design of eddy current probes and the examination of conductive material.

\section{References}

[1] S. Udpa and P. Moore, Nondestructive Testing Handbook, 3 ed., vol. 5, American Nondestructive Society Press, 2004.

[2] Z. Mottl, "The quantitative relations between true and standard depth of penetration for aircored probe coils in eddy current testing," NDT International, vol. 23, pp. 11-18, 1990.

[3] C. Multiphysiscs, "Comsol Multiphysiscs User's Guide," 2010.

[4] S. Majidnia, C. Schneider, J. Rudlin and R. Nilavalan, "A Method of Determining the Eddy Current Instrument Response from Models," Insight: Non-Destructive Testing and Condition Monitoring Journal, vol. 56, pp. 82-86, 2014.

[5] Z. Abidin, Modelling and Experimental Investigation of Eddy Current Distribution for Angular Defect Characterisation, School of Electrical, Electronic and Computer 
Engineering: PhD Thesis, 2010.

[6] Y. He, M. Pan, F. Luo and G. Tian, "Reduction of Lift-Off Effects in Pulsed Eddy Current for Defect Classification," IEEE Transaction on Magnetics, vol. 47, no. 12, pp. 4753-4760, 2011.

[7] A. Sophian, Characterisation of Surface and Sub-Surface Discountinuities in Materials Using Pulsed Eddy Current Sensors, PhD Thesis, 2003, pp. $46-51$. 